\title{
Editorial
}

\section{Feed production: A collaborative challenge}

Food production is the activity with greatest impact on the planet and those who inhabit it. We already produce enough to feed the world, but overconsumption, inequality, waste, and inadequate production and distribution systems stand in the way of having enough food for everyone and space for wildlife. To feed the world in an equitable and environmentally sustainable way, we need to consume and generate livestock products differently. Currently, about $40 \%$ of the usable land surface of the planet is destined to feed its population, most indirectly, since is destined for the feeding of birds, pigs and cattle. The need for land available for livestock production significantly alters ecosystems, causing the extinction of a significant number of species.

The impact of animal feed production does not only affects climate or biodiversity. The appearance of "superbugs" due to antibiotic resistance, in whose generation the livestock production has had a relevant impact, is a serious threat to public health. Approximately 700,000 people worldwide die from infections caused by resistant microorganisms, and it is estimated that by 2050 this number will increase to 10 million per year. Only in the United States, $80 \%$ of antibiotics are not destined for human consumption, they are administered to birds, pigs, fish and cows that are destined for human consumption. In Chile it is estimated that the use of antibiotics is approximately 270 tons per year, and by 2030 this value will increase by $39 \%$. The use of antimicrobials in animals destined for food could be reduced by 2030 between 9 and $80 \%$ with effective policies. This could be achieved by reducing the amount of antimicrobials used per animal or the number of animals we raise for food.

Inequality, overconsumption of protein, reduction of biodiversity, over-demand of ecosystem services and over-use of antibiotics are matters in need of bold public policies. Many of these problems have already been addressed by WHO, FAO and OIE, however, we must ensure that the scope of these measures permeates to producers and professionals related to livestock production. It is important that these guidelines will be implemented considering local idiosyncrasies. Therefore, the role of research institutions, politicians and informed empowered citizens should be focused into addressing these problems in an innovative and collaborative manner.

Editorial Committee Austral Journal of Veterinary Sciences 